\title{
Hairy cell leukemia presenting as a palpable breast mass
}

\author{
E. A. Morgan • L. E. Katzman • D. Georgian-Smith • \\ R. A. Owings • G. S. Pinkus • D. J. DeAngelo
}

Received: 30 June 2014 / Accepted: 1 September 2014 / Published online: 11 September 2014

(C) Springer-Verlag Berlin Heidelberg 2014

\begin{abstract}
Hairy cell leukemia (HCL) is a rare mature B cell malignancy that typically involves the bone marrow, peripheral blood, and spleen. Clinically detectable lymph node infiltration occurs in a minority of patients, and extranodal tissue involvement of sites such as the central nervous system, gastrointestinal tract, and skin is uncommon. Consequently, the vast majority of patients with HCL present with pancytopenia and splenomegaly but without lymphadenopathy or soft tissue masses. Herein we present a unique case of HCL presenting as a palpable breast mass without concomitant marrow or peripheral blood involvement and without radiologic evidence of other sites of disease. Several features of the tumor cell infiltrate (ample pale cytoplasm, nested appearance) evoked other entities that would more typically involve breast tissue such as the alveolar or solid variants of invasive lobular carcinoma. However, the immunophenotypic and molecular characteristics of the tumor cells were indistinguishable from classic HCL. The significant challenge in this case was recognizing this entity outside of its usual clinical and anatomic context. Although rare, HCL should be a diagnostic
\end{abstract}

E. A. Morgan $(\bowtie) \cdot$ R. A. Owings $\cdot$ G. S. Pinkus

Department of Pathology, Brigham and Women's Hospital, Harvard

Medical School, 75 Francis Street, Amory 3, Boston, MA 02115,

USA

e-mail: eamorgan@partners.org

L. E. Katzman

Department of Medicine, Brigham and Women's Hospital, Harvard Medical School, Boston, MA, USA

D. Georgian-Smith

Department of Radiology, Brigham and Women's Hospital, Harvard

Medical School, Boston, MA, USA

D. J. DeAngelo

Department of Medical Oncology, Dana-Farber Cancer Institute, Harvard Medical School, Boston, MA, USA consideration in tumors arising at extramedullary/extranodal sites including the breast.

Keywords Hairy cell leukemia $\cdot$ Extramedullary $\cdot$ Breast . Extranodal

\section{Introduction}

Hairy cell leukemia (HCL) is a rare hematopoietic malignancy of mature B cells, affecting approximately $800-1,000$ patients within the USA annually. The average age at presentation is 50 years with a male/female ratio of 5:1 [1]. HCL typically involves the bone marrow, peripheral blood, and spleen, and as a result patients classically present with cytopenias (including marked monocytopenia) and splenomegaly. SEER database analysis has demonstrated improved survival of patients with HCL over the past three decades attributed to improvements in therapeutic options including alpha interferon and the purine nucleoside analogues pentostatin and cladribine, and life expectancy is near-normal with treatment $[2,3]$. More recently, BRAF V600E mutations have been detected in the great majority of patients with $\mathrm{HCL}$, and initial investigations have demonstrated efficacy of the BRAF kinase inhibitor vemurafenib in patients with relapsed or refractory disease $[4,5]$.

The diagnosis of HCL using a combination of morphologic and immunophenotypic findings in peripheral blood and/or bone marrow is often straightforward. The neoplastic cells of HCL demonstrate strikingly distinct morphologic features, including irregular bean-shaped nuclear contours, ample pale cytoplasm, and circumferential "hairy" cytoplasmic projections best appreciated on smear preparations. The cells express pan-B cell markers such as CD20 and characteristically express CD103, CD25, CD11c, TRAP, DBA.44, annexin A1, CD123, cyclin D1, 
T-bet, and CD200 [6-9]. Additionally, CD10 expression is detected in up to $15 \%$ of cases although expression of this marker does not appear to impact clinical features such as site of involvement or outcome [10], and CD43 expression is only rarely reported in HCL. Testing for the presence of a BRAF V600E mutation can be a useful diagnostic adjunct in challenging cases, as the mutation has not been detected in clinically or pathologically similar disorders such as hairy cell leukemia-variant or splenic marginal zone lymphoma.

Computed tomography (CT) scan studies suggest that lymph node involvement by HCL may occur in up to $15 \%$ of patients, whereas reports of involvement of extranodal tissues such as the skin, central nervous system, or gastrointestinal tract are uncommon [11]. Nodal and extranodal diseases generally arise in patients with concurrent bone marrow or peripheral blood involvement. Herein we present, to our knowledge, the first reported case of HCL presenting as a palpable mass in the breast parenchyma without clinical, radiologic, or pathologic evidence of other sites of involvement.

\section{Case history}

The patient was a 49-year-old woman without a significant past medical history who presented with a palpable breast mass in the upper outer quadrant of the left breast. Per patient report, the mass had been present for nearly 2 years, was non-tender, and had not changed in size. A mammogram performed at an outside institution at the time of self-detection was reportedly read as unremarkable. The patient chose not to pursue further medical attention until approximately 2 years later when establishing care with a new primary care physician. Physical exam revealed a palpable 2-cm mass in the left breast at 12-1 o'clock, no masses in the right breast, and no axillary adenopathy. The patient was otherwise asymptomatic. A CBC performed at that time was unremarkable, demonstrating a white blood cell count of $6.1 \mathrm{~K} / \mathrm{uL}$, hematocrit of $38 \%$, and a platelet count of $302 \mathrm{~K} / \mathrm{uL}$. A differential count was not performed. The patient's family history was notable for breast cancer in her paternal grandmother as well as in her mother, who died of the disease at age 60 .

A mammogram of the left breast revealed heterogeneously dense breast tissue with a round mass with indistinct margins in the upper outer quadrant (Fig. 1a). Concurrent targeted ultrasound demonstrated a solid mass measuring 1.7 $\times 1.7 \times 1.3 \mathrm{~cm}$ in the upper outer quadrant of the left breast, highly suggestive of malignancy (Fig. 1b). Notable ultrasound findings included a hypoechoic center and hyperechoic rim, which is in contrast to a normal lymph node in which the center is hyperechoic (due to fat) and the rim is hypoechoic (due to lymphoid cells in the cortex). Imaging of the right breast was unremarkable. A core needle biopsy was performed.

\section{Materials and methods}

The breast tissue core needle biopsy was fixed in $10 \%$ neutral-buffered formalin. A subsequent bone marrow biopsy was fixed in Bouin solution (StatLab Medical Products, McKinney, TX). Immunohistochemical analysis was performed on paraffin sections as outlined in Table 1. For BRAF V600E mutational analysis, genomic DNA was extracted from formalin-fixed, paraffin-embedded tissue, allele-specific PCR was performed with primers for exon 15 of the $B R A F$ gene, and pyrosequencing was performed using a Qiagen kit with
Fig. 1 Upper outer quadrant left breast mass imaging studies. (a) Mammogram (craniocaudal view) demonstrates a round mass with indistinct margins (large arrow) directly below the skin marker placed at the side of clinical concern (small arrow). There is no architectural distortion or calcifications. (b) Ultrasound evaluation reveals mixed echogenicity with inner hypoechoic and outer hyperechoic regions with this round, circumscribed mass (arrows)

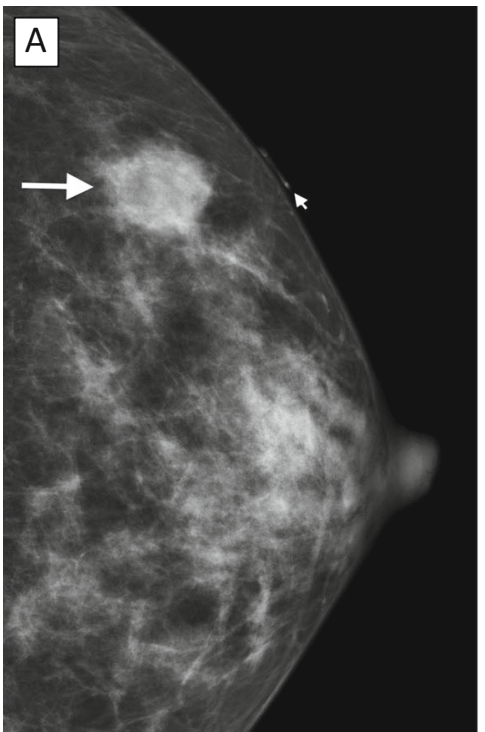


Table 1 Markers evaluated in immunohistochemical analysis

\begin{tabular}{|c|c|c|c|c|c|}
\hline Antigen & Antibody clone & Source $^{\mathrm{a}}$ & Titer & Retrieval & Detection system \\
\hline CD3 & SP7 & Lab Vision & $1: 300$ & $\mathrm{TUF}^{\mathrm{b}} /$ steamer & Rabbit Power Vision (Leica) \\
\hline CD5 & $4 \mathrm{C} 7$ & Leica & $1: 400$ & HIER $\mathrm{pH} 9^{\mathrm{c}}$ & Bond III immunostainer ${ }^{\mathrm{d}}$ \\
\hline Annexin A1 & MRQ-3 & Cell Marque & $1: 100$ & EDTA/steamer & Mouse Power Vision (Leica) \\
\hline CD103 & EPR4166 [2] & Epitomics & $1: 3,000$ & EDTA/steamer & Rabbit Power Vision \\
\hline $\mathrm{CD} 20$ & L26 & Dako & $1: 500$ & EDTA (Ventana) & $\begin{array}{l}\text { Ultraview Universal } \\
\text { DAB Detection } \\
\text { (Ventana immunostainer) }\end{array}$ \\
\hline TRAP & 26E5 & Lab Vision & $1: 40$ & ETDA/steamer & Mouse Power Vision \\
\hline DBA.44 & DBA.44 & Dako & $1: 50$ & TUF/steamer & Mouse Power Vision \\
\hline $\mathrm{CD} 25$ & $4 \mathrm{C} 9$ & Vector & $1: 200$ & EDTA/steamer & Mouse Power Vision \\
\hline CD123 & $9 \mathrm{~F} 5$ & BD Biosciences & $1: 25$ & EDTA/steamer & Mouse Power Vision \\
\hline Cyclin D1 & SP4 & Thermo Scientific & $1: 30$ & HIER pH 9 & Bond III immunostainer \\
\hline CD10 & $56 \mathrm{C} 6$ & Leica & $1: 80$ & HIER pH 9 & Bond III immunostainer \\
\hline CD43 & Leu-22 & BD Biosciences & $1: 10,000$ & EDTA/steamer & Mouse Power Vision \\
\hline $\mathrm{CD} 23$ & $1 \mathrm{~B} 12$ & Cell Marque & $1: 50$ & Pressure cooker/Dako target retrieval & Mouse Power Vision \\
\hline MPO & Rabbit poly & Dako & $1: 25,000$ & None & Rabbit Power Vision \\
\hline Keratin & AE1/AE3 & Dako & $1: 200$ & EDTA/steamer & Mouse Power Vision \\
\hline CD34 & QBend-10 & Beckman Coulter & $1: 70$ & Pressure cooker/citrate $\mathrm{pH} 6$ & Mouse Power Vision \\
\hline S100 & Rabbit poly & Dako & $1: 1,000$ & None & Envision $+(\text { rabbit, } \mathrm{DAB})^{\mathrm{e}}$ \\
\hline Ki67 & MIB-1 & Dako & $1: 500$ & HIER pH 9 & Bond III immunostainer \\
\hline$B R A F \mathrm{~V} 600 \mathrm{E}$ & VE1 & Spring Bioscience & $1: 60$ & HIER pH 9 & Bond III immunostainer \\
\hline ER & SP1 & Thermo Scientific & $1: 40$ & Citrate/pressure cooker & Envision+ \\
\hline PR & PgR636 & Dako & $1: 150$ & Citrate/pressure cooker & Envision+ \\
\hline HER2neu & SP3 & Thermo Scientific & $1: 50$ & Citrate/pressure cooker & Envision+ \\
\hline
\end{tabular}

EDTA ethylenediaminetetraacetic acid, $\mathrm{pH}$ 8.0; HIER heat-induced epitope retrieval

${ }^{a}$ Lab Vision Corporation (Fremont, CA), Leica Biosystems, Inc. (Buffalo Grove, IL), Cell Marque (Rocklin, CA), Epitomics (Burlingame, CA), Dako (Carpinteria, CA), Vector Laboratories, Inc. (Burlingame, CA), BD Biosciences (San Jose, CA), Thermo Scientific (Waltham, MA), Beckman Coulter, Inc. (Miami, FL), Spring Bioscience (Pleasanton, CA), Ventana Medical Systems (Tucson, AZ)

${ }^{\mathrm{b}}$ Target Unmasking Fluid, Life Technologies (Carlsbad, CA)

${ }^{\mathrm{c}}$ Bond reagents, Leica Biosystems, Inc.

${ }^{\mathrm{d}}$ Bond Polymer Refine DAB Detection

${ }^{\mathrm{e}}$ Dako

primers for codons 559 to 601 . The resulting sequence was compared to a wild-type control.

\section{Results}

Microscopic examination of the biopsy revealed almost complete effacement of normal breast parenchyma by a diffuse infiltrate of small-sized cells with round to bean-shaped nuclear contours, condensed chromatin, inconspicuous nucleoli, and ample pale cytoplasm (Fig. 2a, b). In areas, the cells were surrounded by delicate bands of fibrosis, imparting a nested appearance. Few small foci of residual breast glandular tissue were present in one core fragment (Fig. 2c). No nodal tissue was appreciated on multiple levels. Several small aggregates of unremarkable lymphocytes were interspersed. There was no morphologic evidence of a primary breast carcinoma.

Immunohistochemical studies revealed that the infiltrate was composed of CD20-positive B cells which expressed LCA, annexin A1, CD103 (subset), TRAP, DBA.44, CD25, CD123, cyclin D1, CD10, and CD43 (Fig. 2d-g). Antibodies to CD3, CD5, CD23, myeloperoxidase, AE1/AE3, CD34, S100, ER, PR, and HER2neu were non-reactive in the infiltrate. The Ki67 proliferation index was less than $5 \%$. A $B R A F$ V600E mutation-specific antibody exhibited cytoplasmic reactivity in the lesional cells (Fig. $2 \mathrm{~h}$ ). A reticulin stain highlighted delicate reticulin fibrosis throughout the infiltrate (Fig. 2i). The few small aggregates of unremarkable lymphocytes were composed of CD3-positive $\mathrm{T}$ cells which appropriately co-expressed CD5 and CD43. Molecular 


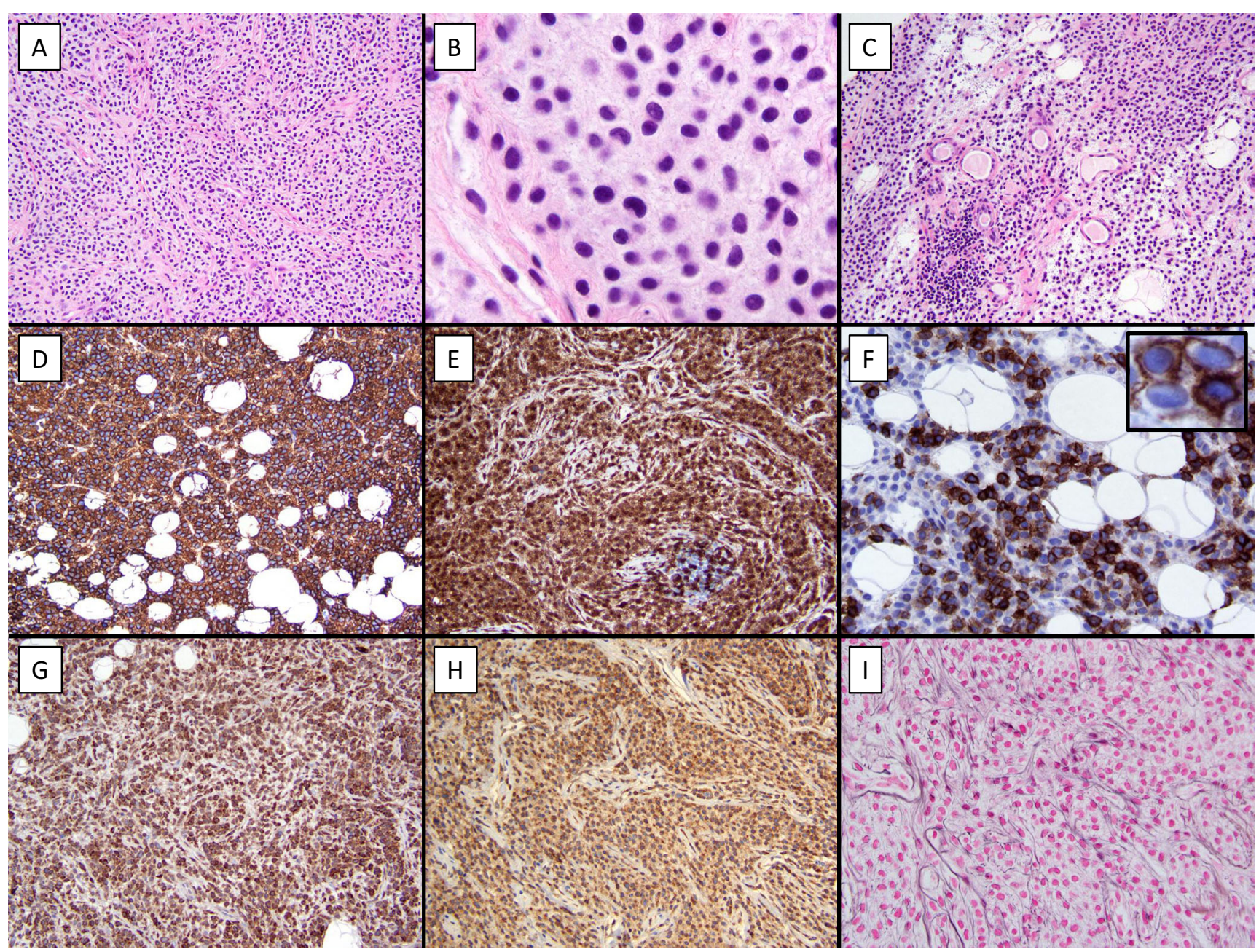

Fig. 2 Breast tissue involvement by HCL. (a) Low $(\times 200)$ and (b) high $(\times 1,000)$ magnification views of HCL (hematslin and eosin, $\mathrm{H} \& \mathrm{E}$ ). (c) The HCL cells directly infiltrate breast parenchyma $(\times 200, \mathrm{H} \& \mathrm{E})$. HCL tumor cells express CD20 (d), annexin A1 (e), CD103 (f), and

analysis revealed the presence of the $B R A F \mathrm{~V} 600 \mathrm{E}$ mutation as well as a clonal immunoglobulin heavy chain gene rearrangement. A diagnosis of $\mathrm{HCL}$ was rendered.

Following the diagnosis, a repeat $\mathrm{CBC}$ with differential was obtained with all values within the reference ranges including the absolute monocyte count. CT scans of the chest, abdomen, and pelvis were unremarkable except for the previously noted left breast mass. Pertinent negatives included no evidence of lymphadenopathy or splenomegaly. Magnetic resonance imagining of the left breast was also unremarkable except for the previously noted mass. A bone marrow biopsy was performed which revealed a mildly hypocellular for age marrow ( $30 \%$ cellular) with maturing trilineage hematopoiesis and no evidence of involvement by HCL by immunophenotyping (not shown). Flow cytometric analysis did not reveal an atypical B cell population. The karyotype was normal (46, XX [20]). Molecular analysis revealed only wild-type $B R A F$ sequences. A conservative "watch and wait" approach to therapy was recommended.
TRAP (g) and demonstrate reactivity with the BRAF V600E mutationspecific antibody $(h)(\mathrm{d}, \mathrm{e}, \mathrm{g}, \mathrm{h} \times 200 ; \mathrm{f} \times 400$; inset $\times 1,000)$. (i) A reticulin stain highlights delicate interspersed fibrosis $(\times 200)$

\section{Discussion}

To our knowledge, this is the first reported case of HCL presenting as a palpable breast mass without clinical, radiologic, or pathologic evidence of other sites of involvement. Although the tumor conformed to the standard morphologic, immunophenotypic, and molecular criteria for HCL, this case is remarkable for both the atypical clinical presentation and unusual pattern of disease involvement. The significant challenge in this case was recognizing this entity outside of its usual context and initiating the appropriate diagnostic work-up.

HCL is an indolent neoplasm of small B cells that typically involves the peripheral blood, bone marrow, and spleen. As a result of this pattern of involvement, patients often present with fatigue, bleeding, or infections secondary to cytopenias, as well as left upper quadrant pain or early satiety due to splenomegaly. Monocytopenia is frequently observed. Other signs and symptoms of the disease due to 
extramedullary tissue involvement are unusual. For example, while HCL cells are typically present in the liver sinusoids at the time of diagnosis, clinically evident hepatic manifestations such as hepatomegaly are infrequent [11]. Lymphadenopathy is an uncommon presenting symptom, and estimates from CT scan-based studies suggest that nodal involvement is seen in a minority of patients with established HCL (up to $15 \%$ ) [11]. Infrequently, massive retroperitoneal lymphadenopathy occurs in the setting of long-standing disease and portends a poor prognosis [12]. Extranodal tissue involvement in HCL is also uncommon, although many sites have been reported in autopsy and single/small case series including ascitic fluid, central nervous system, gastrointestinal tract, kidney, lungs/pleural space, pericardium, peripancreatic soft tissue, skeleton, and skin [11, 13-20].

HCL involving breast parenchyma is exceeding rare, with only one case reported in the literature [21]. Indeed, a comprehensive review of 106 patients with lymphoma involving the breast seen at a major referral center did not document any cases of HCL [22]. In comparing our report with that of Farkash et al., several differences are apparent. While both women presented with a breast mass, microscopic examination of the breast tissue from the patient reported by Farkash et al. demonstrated extensive ductal carcinoma in situ in addition to an infiltrate of HCL cells. Therefore, it is uncertain to what degree, if any, the HCL infiltrate contributed to the development of the mammographically detectable breast mass. In addition, concurrent work-up of the patient reported by Farkash et al. revealed evidence of bone marrow, skeletal, and lymph node involvement by HCL, as well as mild anemia and absolute monocytopenia. These findings prompted therapy with cladribine. In contrast, our patient exhibited no evidence of disease outside of the breast by clinical, radiologic, pathologic, and molecular investigation.

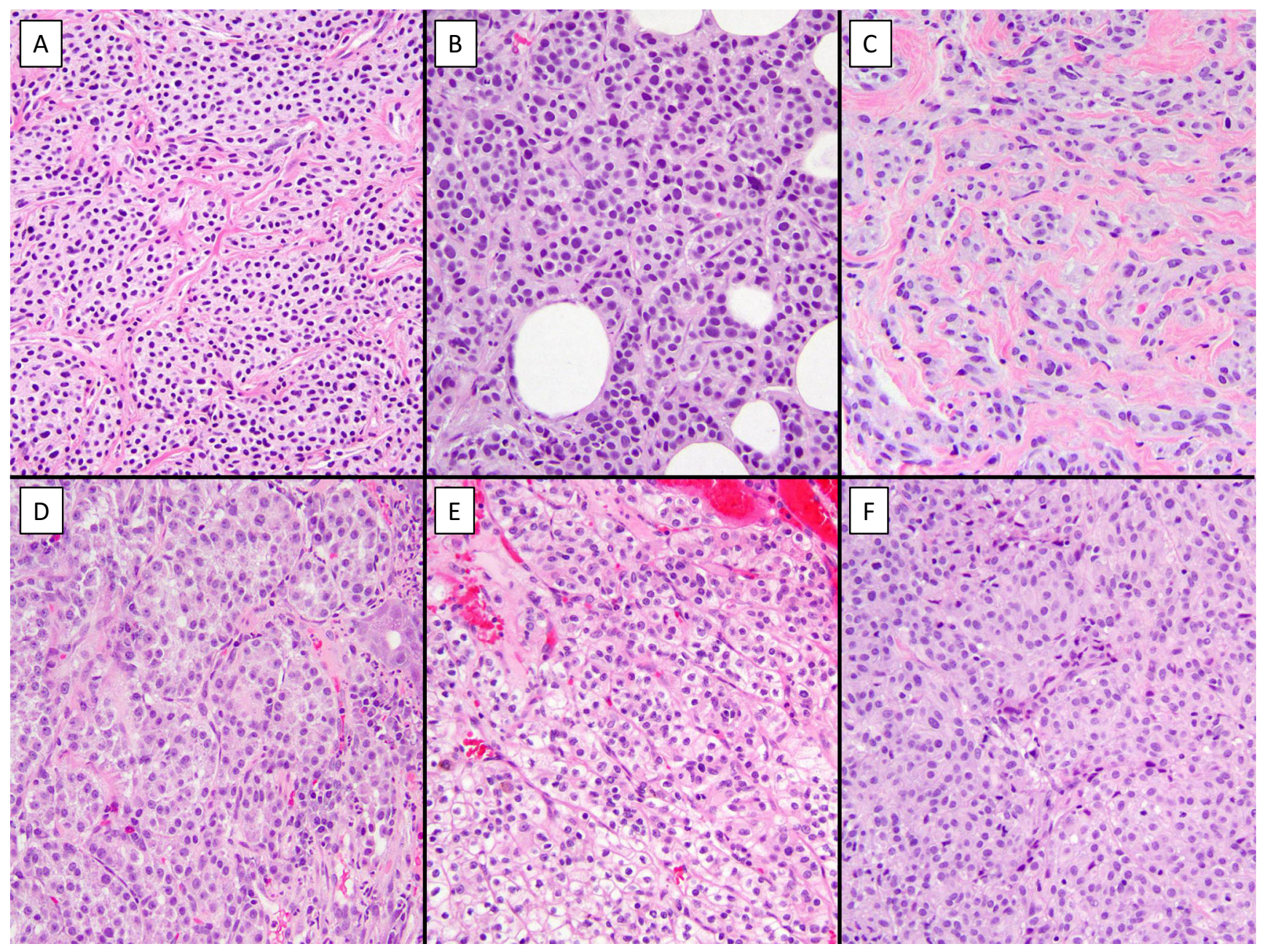

Fig. 3 Morphologic differential diagnosis for extranodal HCL. Several non-hematopoietic entities share the epithelioid appearance and vaguely nested pattern of infiltration of HCL (a). Diagnostic considerations of neoplasms involving breast parenchyma include the solid variant of invasive lobular carcinoma (b) or mammary-type myofibroblastoma, epithelioid variant (c). Outside of the breast, other neoplastic entities demonstrating overlapping morphologic features with HCL include well-differentiated neuroendocrine tumors (d), clear cell renal cell carcinoma (e), or epithelioid gastrointestinal stromal sarcoma (f) (all H \& E, all $\times 200$ ) 
This was a remarkable finding given that the vast majority of reported cases of extranodal HCL have occurred in patients with established or concomitant HCL. In the absence of other sites of disease or other indication for treatment such as symptomatic splenomegaly, symptoms of bone marrow failure, or decline in peripheral blood counts, a watch and wait approach was recommended for our patient [2]. In the year following the diagnosis, peripheral blood counts remain within normal limits, and there is no evidence of splenomegaly. In addition, the size of the breast mass has remained stable for nearly 3 years.

Extramedullary/extranodal involvement by HCL can pose a diagnostic challenge, particularly to pathologists who do not routinely encounter hematopathology specimens. Although the morphologic appearance of hairy cells is readily recognizable with experience, several histologic features out of the context of a hematopoietic organ may be confounding. The excess amount of cytoplasm in HCL cells imparts an epithelioid appearance which is atypical for a lymphocyte (Fig. 3a). Further, the nested configuration of the cells, likely due to associated reticulin fibrosis, stands in contrast to the typical nodular or diffuse pattern of extranodal lymphomatous involvement and is more reminiscent of a non-hematopoietic neoplasm. Within the breast parenchyma, diagnostic considerations based on the morphologic appearance would include the alveolar or solid variants of invasive lobular carcinoma (Fig. 3b) [21] or mammary-type myofibroblastoma, epithelioid variant (Fig. 3c). In terms of any extramedullary/ extranodal site, other morphologic mimics include welldifferentiated neuroendocrine tumors (Fig. 3d), low-grade clear cell renal cell carcinoma (Fig. 3e), epithelioid soft tissue neoplasms (Fig. 3f), or melanocytic proliferations. In general, the nuclei of HCL cells are smaller and exhibit more condensed chromatin than these other entities, and the cytoplasm appears less amphophilic. In this case, if hairy cell leukemia had not been suspected upon morphologic grounds, the absence of reactivity for ER, PR, and HER2neu in conjunction with low-grade morphology would have prompted suspicion that the neoplasm was not a primary breast carcinoma.

Recent advances in the diagnosis of HCL permitted confident diagnosis in this unusual case. The proven specificity of annexin A1 for HCL among small B cell lymphomas is useful for distinction of HCL from other similar hematopoietic neoplasms such as hairy cell leukemiavariant and splenic marginal zone lymphoma [23]. In addition, the recent validation of an antibody to CD103 for use in fixed, paraffin-embedded tissues permitted evaluation of CD103 expression in this specimen for which (understandably) concurrent flow cytometric analysis was not performed [24]. Finally, the discovery of $B R A F$ $\mathrm{V} 600 \mathrm{E}$ mutations in nearly all cases of HCL provided an opportunity for confirmatory molecular analysis [4].

In summary, we present a unique case of HCL presenting as a palpable breast mass without clinical, radiologic, or pathologic evidence of disease at other anatomic sites. The diagnosis required recognition of this entity in an unusual clinical context and anatomic location, and confirmation relied upon a combination of characteristic morphologic, immunophenotypic, and molecular features. Although uncommon, HCL should be included as a diagnostic consideration in tumors involving extramedullary/extranodal sites including the breast.

Conflicts of interest The authors declare that they have no conflict of interest. There are no disclosures/funding sources to report.

\section{References}

1. Else M, Ruchlemer R, Osuji N, Del Giudice I, Matutes E, Woodman A et al (2005) Long remissions in hairy cell leukemia with purine analogs: a report of 219 patients with a median follow-up of 12.5 years. Cancer 104(11):2442-2448

2. Grever MR (2010) How I treat hairy cell leukemia. Blood 115(1):2128

3. Chandran R, Gardiner SK, Smith SD, Spurgeon SE (2013) Improved survival in hairy cell leukaemia over three decades: a SEER database analysis of prognostic factors. Br J Haematol 163(3):407-409

4. Tiacci E, Trifonov V, Schiavoni G, Holmes A, Kern W, Martelli MP et al (2011) BRAF mutations in hairy-cell leukemia. N Engl J Med 364(24):2305-2315

5. Dietrich S, Glimm H, Andrulis M, von Kalle C, Ho AD, Zenz T (2012) BRAF inhibition in refractory hairy-cell leukemia. N Engl J Med 366(21):2038-2040

6. Sherman MJ, Hanson CA, Hoyer JD (2011) An assessment of the usefulness of immunohistochemical stains in the diagnosis of hairy cell leukemia. Am J Clin Pathol 136(3):390-399

7. Venkataraman G, Aguhar C, Kreitman RJ, Yuan CM, StetlerStevenson M (2011) Characteristic CD103 and CD123 expression pattern defines hairy cell leukemia: usefulness of CD123 and CD103 in the diagnosis of mature B-cell lymphoproliferative disorders. Am J Clin Pathol 136(4):625-630

8. Dorfman DM, Hwang ES, Shahsafaei A, Glimcher LH (2004) T-bet, a T-cell-associated transcription factor, is expressed in a subset of Bcell lymphoproliferative disorders. Am J Clin Pathol 122(2):292-297

9. Dorfman DM, Shahsafaei A (2010) CD200 (OX-2 membrane glycoprotein) expression in B cell-derived neoplasms. Am J Clin Pathol 134(5):726-733

10. Chen YH, Tallman MS, Goolsby C, Peterson L (2006) Immunophenotypic variations in hairy cell leukemia. Am J Clin Pathol 125(2):251-259

11. Tadmor T, Polliack A (2011) Unusual clinical manifestations, rare sites of involvement, and the association of other disorders with hairy cell leukemia. Leuk Lymphoma 52(Suppl 2):57-61

12. Shackelford RE, Heldmann M, Eskandari F, Joshi N, Browning J, Maxwell N et al (2013) Marked retroperitoneal lymphadenopathy in hairy cell leukemia: a case report. Case Rep Oncol 6(3): 493-496

13. Vardiman JW, Variakojis D, Golomb HM (1979) Hairy cell leukemia: an autopsy study. Cancer 43(4):1339-1349

14. Vardiman JW, Golomb HM (1984) Autopsy findings in hairy cell leukemia. Semin Oncol 11(4):370-380

15. Bouroncle BA (1987) Unusual presentations and complications of hairy cell leukemia. Leukemia 1(4):288-293 
16. Dedic K (2003) Hairy cell leukemia: an autopsy study. Acta Med (Hradec Kralove) 46(4):175-177

17. Ergene U, Ozbalci D, Isisag A (2012) Hairy cell leukemia and cutaneous involvement. Transfus Apher Sci 46(2):231-232

18. Subhawong AP, Subhawong TK, Ali SZ (2012) Hairy cell leukemia presenting as a peripancreatic mass: cytomorphology and radiographic correlates. Acta Cytol 56(4):463-466

19. Osman H, Roth T, Zhou J (2013) Hairy cell lymphoma: a unique presentation of hairy cell leukaemia. Br J Haematol 162(3):294

20. Chandana SR, Kotecha R, Al-Janadi A, Chang HT, Conley BA (2013) Rare case of hairy cell leukemia with brain parenchymal involvement: a diagnostic dilemma. J Clin Oncol 31(11):e186-8

21. Farkash EA, Ferry JA, Harris NL, Hochberg EP, Takvorian RW, Zuckerman DS et al (2009) Rare lymphoid malignancies of the breast: a report of two cases illustrating potential diagnostic pitfalls. J Hematop 2(4):237-244

22. Talwalkar SS, Miranda RN, Valbuena JR, Routbort MJ, Martin AW, Medeiros LJ (2008) Lymphomas involving the breast: a study of 106 cases comparing localized and disseminated neoplasms. Am J Surg Pathol 32(9):1299-1309

23. Falini B, Tiacci E, Liso A, Basso K, Sabattini E, Pacini R et al (2004) Simple diagnostic assay for hairy cell leukaemia by immunocytochemical detection of annexin A1 (ANXA1). Lancet 363(9424): $1869-1870$

24. Morgan EA, Yu H, Pinkus JL, Pinkus GS (2013) Immunohistochemical detection of hairy cell leukemia in paraffin sections using a highly effective CD103 rabbit monoclonal antibody. Am J Clin Pathol 139(2):220-230 